\title{
Changes on the nanostructure of cementitius calcium silicate hydrates (C-S-H) induced by aqueous carbonation.
}

Morales-Florez, V. ${ }^{1,2}$, Findling, N. ${ }^{2,3}$, Brunet, F. ${ }^{2,3}$

${ }^{1}$ Instituto de Ciencia de Materiales de Sevilla (CSIC-US). Centro de Investigaciones Científicas Isla de la Cartuja, 41092 Seville, Spain.

${ }^{2}$ Laboratoire de Géologie. Ecole normale supérieure - CNRS. 75231 Paris Cedex 05, France.

${ }^{3}$ Institut des Sciences de la Terre, CNRS - Université Joseph Fourier. 38041 Grenoble Cedex 9, France

\section{DOI 10.1007/s10853-011-5852-6}

\section{Abstract}

The nanostructure of the main binding phase of the hydrated cements, the calcium silicate hydrates (C-S-H), and their structural changes due to aqueous carbonation have been characterized using TEM, nitrogen physisorption and SAXS. Synthetic C-S-H has been used for this purpose. Two different morphologies were identified, similar to the high density and low density C-S-H types. When submitting the sample to a $\mathrm{CO}_{2}$ flux, the low density phase was completely carbonated. The carbonation by-products, calcium carbonate and silica gel were also identified and characterized. The precipitation of the silica gel increased the specific surface area from $95 \mathrm{~m}^{2} / \mathrm{g}$ to $132 \mathrm{~m}^{2} / \mathrm{g}$, and its structure, formed by particles of $\sim 5 \mathrm{~nm}$ typical radius was observed by small angle $\mathrm{x}$-ray scattering. In addition, the resistance of the high density $\mathrm{C}-\mathrm{S}-\mathrm{H}$ to carbonation is reported and the passivating effect of the precipitated calcium carbonate is also discussed. Finally, the results have been compared with carbonation features observed in Portland cement 
carbonated experimentally at downhole conditions.

KEYWORDS: Calcium-Silicate-Hydrate (C-S-H); Small-Angle X-Ray Scattering; TEM; Pore Size Distribution; Surface Area

\section{Introduction}

Calcium silicate hydrate $(\mathrm{C}-\mathrm{S}-\mathrm{H})^{*}$ is the main binding phase of hydrated cements [1] and is formed by the hydration of the $\mathrm{C}_{2} \mathrm{~S}$ and $\mathrm{C}_{3} \mathrm{~S}$ phases of the cement. It is a porous particulate quasi-mineral of variable composition which plays a central role in the properties of hydrated cements, such as mechanical properties, diffusivity or durability of most of the constructions. Specifically, the reactivity of hydrated cements with carbon dioxide has become a topic of major importance given the current expansion of the technology of carbon removal by geological storage. This technology is already being implemented to reduce the anthropological emissions of greenhouse gases to the atmosphere, and carbon dioxide injection wells are being sealed with cement [2,3]. The reactivity and characteristics of hydrated cements in contact with $\mathrm{CO}_{2}$ is currently under research. Among the different calcium-rich phases of the cement, the C-S-H does also carbonate yielding calcium carbonates, silica gel, and water [4-6]. Therefore, knowing the properties of hydrated cements in contact with $\mathrm{CO}_{2}$-rich environments, both supercritical

\footnotetext{
*We use the cement notation: $\mathrm{C}=\mathrm{CaO}, \mathrm{S}=\mathrm{SiO}_{2}$ and $\mathrm{H}=\mathrm{H}_{2} \mathrm{O}$. In the $\mathrm{C}-\mathrm{S}-\mathrm{H}$ notation, the hyphenation indicates variable composition.
} 
$\mathrm{CO}_{2}$ and $\mathrm{CO}_{2}$-rich aqueous fluids, and understanding the structural and chemical changes encountered by $\mathrm{C}-\mathrm{S}-\mathrm{H}$ as main binding phase, is critical to estimate and improve the efficiency and durability of underground $\mathrm{CO}_{2}$ storage.

In the literature, $\mathrm{C}-\mathrm{S}-\mathrm{H}$ is often referred to as a gel although it must rather be considered as a precipitate due to the presence of capillary pores in cement pastes [7]. The structure of $\mathrm{C}-\mathrm{S}-\mathrm{H}$ is very heterogeneous at the nanometric scale, so developing a complete structural model is a complex task. For simplification, C-S-H's are often categorized as types I and II, outer and inner product (OP and IP) or high density (HD) and low density C-S-H (LD) [1,8-10]. This binary description has been established since two different particulate nanostructures can be found in C-S-H's, depending on where they form with respect to the parent anhydrous C-S, namely, in the pore space among the original clinker grains or in the space initially occupied by them. The inner product is a rough and dense disordered particulate matrix, whereas the outer product is a less dense phase with sheet-like and needle-like morphologies. Transmission Electron Microscopy (TEM) imaging clearly illustrates [11] the aforementioned types of C-S-H.

These nanostructures can be described as assemblages of random packings of particles with embedded water molecules [12], arranged in several hierarchical levels [10, 11, 13, 14] that eventually extends through the space between mineral grains [15]. Small angle scattering experiments (SAXS, if $\mathrm{x}$-rays are used as radiation, or SANS if neutrons are used) have been used to research the $\mathrm{C}-\mathrm{S}-\mathrm{H}$ - formation during hydration of the cement, reporting the surface fractal morphology of C-S-H [16] eleven hours after hydration of cement on the length scale $10-100 \mathrm{~nm}$. These experiments have also revealed some characteristic features as the multi-level structures, the density and the role of the water in the basic building blocks $[17,18]$ of $\mathrm{C}-\mathrm{S}-\mathrm{H}$. The size, shape or crystallographic nature and water content of the basic building blocks, and their arrangements forming 
hierarchical fractal structures, are currently the focus of research efforts, both experimentally [19-21] and computationally [14,22]. Tobermorite or jennite are being proposed as structural references for the basic building blocks $[11,23]$.

The structural changes of cementitius calcium-rich phases due to carbonation have previously been studied. For example [24], it has been shown that $\sim 50 \mathrm{~nm}$ clusters appear under the influence of different environments containing $\mathrm{CO}_{2}$. The carbonation effect on cement mortar has also been studied, pointing out the appearance of pores with radii between $2 \mathrm{~nm}$ and $7 \mathrm{~nm}$, and no change in the specific surface area of the mortar [25]. In order to focus our study on the effect of carbonation on C-S-H structural properties, C-S-H was synthesized separately, and the effect of carbonation was compared with those obtained on hydrated Portland cements.

\section{Experimental methods}

Sample preparation

The C-S-H sample was synthesized from a solution with a $\mathrm{Ca} / \mathrm{Si}$ ratio of 1.8 according to the co-precipitation method [26] which consists of adding, under stirring, a solution of $\mathrm{Na}_{2} \mathrm{SiO}_{3}($ Merck, $1 \mathrm{l}=1.35 \mathrm{~kg})$ to a calcium saturated solution. This solution was aged for up to 21 days in sealed containers inside an argon-filled glovebox at ambient pressure and temperature. In this inert environment, the supernatant was exchanged with $\mathrm{CO}_{2-}$ free water to remove $\mathrm{Na}$ traces. This washing yielded a $\mathrm{Na}$ content in the final solid phase down to $1 \mathrm{wt}$. \%. Afterwards, the supernatant was removed and the residual solid product was dried inside a glovebox at ambient conditions in the presence of silica gel for 
several weeks. Silica gel was renewed every two days under a vigorous argon flux to minimize air (and therefore $\mathrm{CO}_{2}$ ) contamination. Hereafter, this sample will be referred to as "original C-S-H sample". In addition, a hydrated cement reference sample (Class G well cement, slurry density $=1.89 \mathrm{~g} / \mathrm{cm}^{3}$ ) was also characterized to compare both cement and C-S-H structures. Full details of the cement reference sample preparation, processing, and carbonation can be found in Ref. 4 .

\section{Carbonation experiments}

The original C-S-H sample was dispersed in $\mathrm{CO}_{2}$-saturated water under vigorous stirring in an open Teflon open reactor (at ambient pressure and temperature). Then, a $\mathrm{CO}_{2}$ flux $\left(20 \mathrm{~cm}^{3} / \mathrm{s}, 1.5 \mathrm{bar}\right.$ ) was imposed for 15 minutes, setting the $\mathrm{pH}$ of the aqueous medium down to $\sim 6$. Afterwards, the powder sample rested in the $\mathrm{CO}_{2}$-saturated water overnight and was then dried under atmospheric conditions. This sample will be referred to hereafter as "carbonated C-S-H sample". For comparison purposes, a sample of hydrated cement was cored and carbonated under static conditions at $90^{\circ} \mathrm{C}$ and 280 bars (simulation of downhole conditions) for six months in order to obtain a reference carbonated cement sample. Full details of the carbonation of hydrated cement can be found in Ref. 4.

Characterization techniques

Nitrogen physisorption experiments (Micrometrics, model ASAP2010) were made at a constant temperature of $77.35 \mathrm{~K}$. Samples were milled and degasified at $150^{\circ} \mathrm{C}$ for two hours. Isotherm curves were analyzed by the Brunauer-Emmet-Teller [27] and Barret- 
Joyner-Halenda [28] methods to obtain specific surface area $\left(\mathrm{S}_{\mathrm{BET}}\right)$ and pore size distribution (PSD), respectively. For Transmission Electron Microscopy (TEM), samples were finely milled in an agate mortar and then dispersed in ethanol. Dispersion was assisted by an ultrasound bath. One drop of this dispersion was poured onto the holey carbon sample holder. The TEM images (JEOL 2011 microscope) were taken using an electron acceleration voltage of $200 \mathrm{keV}$, yielding a wavelength of $2.5 \cdot 10^{-3} \mathrm{~nm}$. Image processing was made with the ImageJ $v 1.40 \mathrm{~g}$ software package [29]. The possible beam damage was minimized by limiting the sample exposition to the electron beam to less than $30 \mathrm{~s}$. No damages were observed on the $\mathrm{C}-\mathrm{S}-\mathrm{H}$ phase but damages were found in the calcium carbonate crystals.

Small angle x-ray scattering (SAXS) experiments were performed at the BM16 workstation at the European Synchrotron Radiation Facility (ESRF) in Grenoble (France). Milled powders were enclosed between kapton tapes in the center of metallic washers of $8 \mathrm{~mm}$ inner diameter. Signals from empty metallic washers were measured for background removal. For these experiments, the wavelength of the X-ray beam was $0.098 \mathrm{~nm}$. The SAXS workstation was previously calibrated using a reference sample of silver behenate. The experimental set-up (the CDD detector camera size, beam-stop size, and the maximum detector-to-sample distance of $14 \mathrm{~m}$ ) allowed exploring a $q$ range from $0.05 \mathrm{~nm}^{-1}$ to $1.47 \mathrm{~nm}^{-1}$.

\section{Results and discussion}

Nitrogen physisorption experiments

First, the structure of the C-S-H samples was studied by nitrogen physisorption 
experiments (Figure 1). Type IV curves are typically obtained in mesoporous materials with a hysteresis loop type $\mathrm{H} 2$, indicating irregular pore shape and size distribution [30]. Analyses of the curves yielded the texture data shown in Table 1. In the case of the C-S$\mathrm{H}$ samples (original and carbonated), the results showed an increase of the specific surface area from $95 \mathrm{~m}^{2} / \mathrm{g}$ to $132 \mathrm{~m}^{2} / \mathrm{g}$, and an increase of the specific porous volume from $0.52 \mathrm{~cm}^{3} / \mathrm{g}$ to $0.68 \mathrm{~cm}^{3} / \mathrm{g}$ due to the $\mathrm{CO}_{2}$ attack. Note that large non-porous calcium carbonate crystals coexisted with the porous phases and contributed to the total mass, but their contribution to the specific surface area is negligible. Therefore, the values of the surface and porous volume of the particulate phases are underestimated. The pore size distribution (PSD) did not show a major modification with carbonation, though the carbonated sample showed a wider distribution corresponding to an increased polydispersity of the pores (Figure 1). These features, i.e. the increase in specific surface area and porous volume due to carbonation, can be explained in terms of precipitation of silica gel, a common by-product of the carbonation of the calcium silicate hydrates.

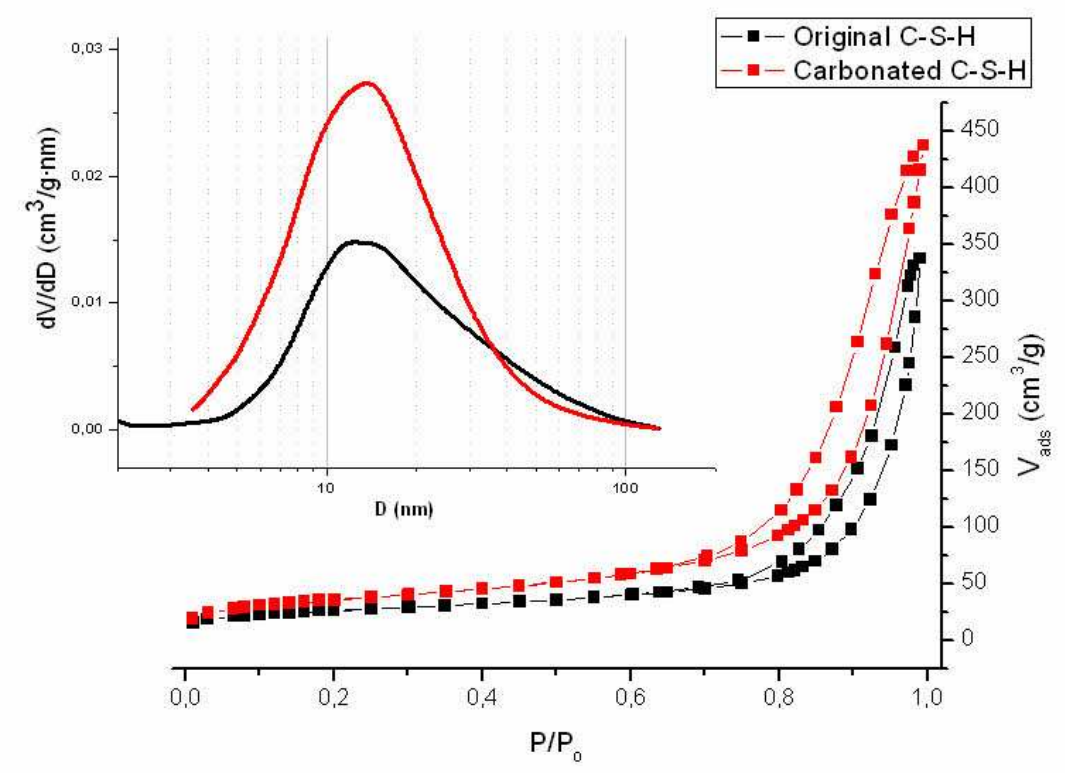

\section{FIGURE 1}




\section{TABLE 1}

In the case of the Portland cement samples, both isotherms display type II curves, corresponding to non-porous or macroporous adsorbents. The carbonated cement presented a hysteresis loop type revealing capillary condensation typically found for silica gel. The specific surface area $\left(26 \mathrm{~m}^{2} / \mathrm{g}\right)$ and specific porous volume $\left(0.07 \mathrm{~cm}^{3} / \mathrm{g}\right)$ remained constant for both carbonated and non carbonated samples, similar to what has been reported with water adsorption [25]. Thus, the precipitation of porous silica gel due to carbonation made up for the disappearance of porous C-S-H. Nevertheless, these values are not representative of the texture values (specific surface area and porous volume) of the C-S-H of the hydrated cement, as this porous phase coexists with other non-porous phases in hardened hydrated cements that contribute to the total sample mass. And again, the PSD of cements broadens with the carbonation process, indicating again higher polydispersity in the pore sizes, given the formation of the silica gel byproduct.

Transmission electron microscopy

The TEM images of the original sample showed randomly oriented needles $\sim 100 \mathrm{~nm}$ in size in a disordered mesoporous matrix (Figure 2), similar to the reported morphology of the LD C-S-H. Looking closer at the disordered phase (Figure 3), embedded nanocrystals of less than $10 \mathrm{~nm}$ could be found. The presence of these nanocrystals was previously reported by Zangh [31] and Viehland [32], who explained that this disordered phase corresponds to HD C-S-H of the cement hydration process. Xu [33] also observed nanometric crystals in hydrated cements that developed very rapidly in several days. So 
in this synthetic C-S-H, a mixture of both LD and HD C-S-H was found. Interplanar distances determined directly on the TEM images or from analyzing the electron diffraction patterns (inset of Figure 3) are shown in Table 2. They were indexed as tobermorite reflections [34]. It was also observed that the needles showed a very poor crystallinity, with interplanar distances of $1.10 \pm 0.1 \mathrm{~nm}$.

\section{TABLE 2}

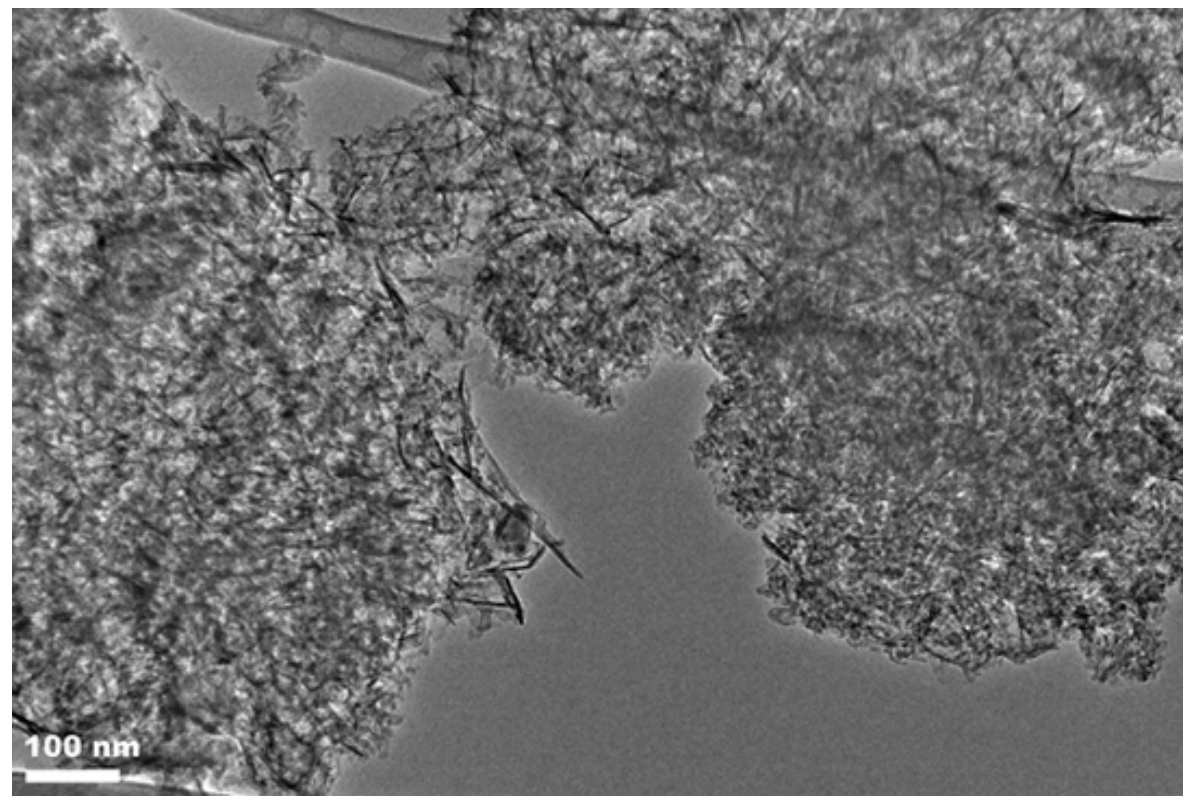

FIGURE 2 


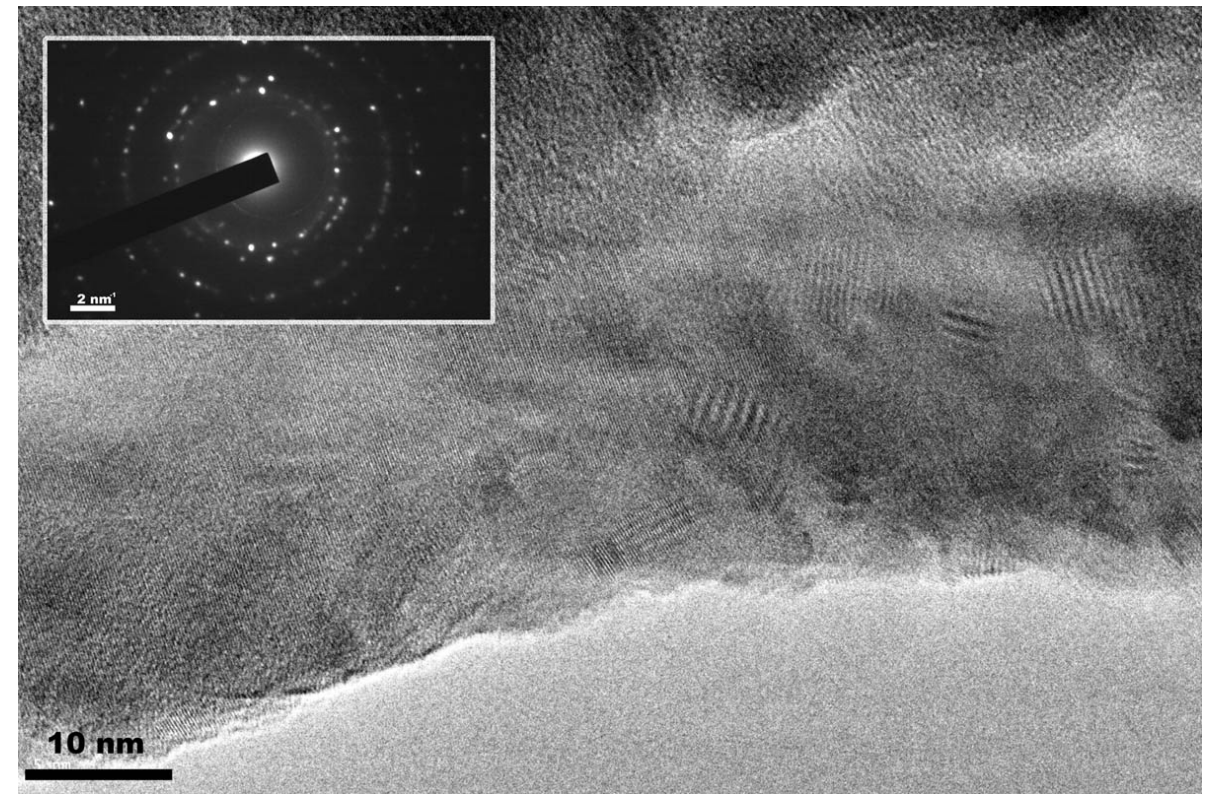

\section{FIGURE 3}

The images of the carbonated C-S-H present a different morphology (Figure 4).

Tobermorite needles are no longer found, but embedded nanocrystals can still be observed in the disordered matrix (lower part of Figure 5), corresponding to remaining HD C-S-H. The reflections on the electron diffraction pattern obtained in that region were identified again as tobermorite from the interplanar distances (Table 2). In Figure 5, the silica by-product (upper right part of Figure 5) can be clearly differentiated from the remaining HD C-S-H. This silica gel shows particle and pore sizes in the order of $13 \mathrm{~nm}$, as indicated by the reference circle of $13 \mathrm{~nm}$ in Figure 5, similar to the pore size observed in nitrogen physisorption experiments. 


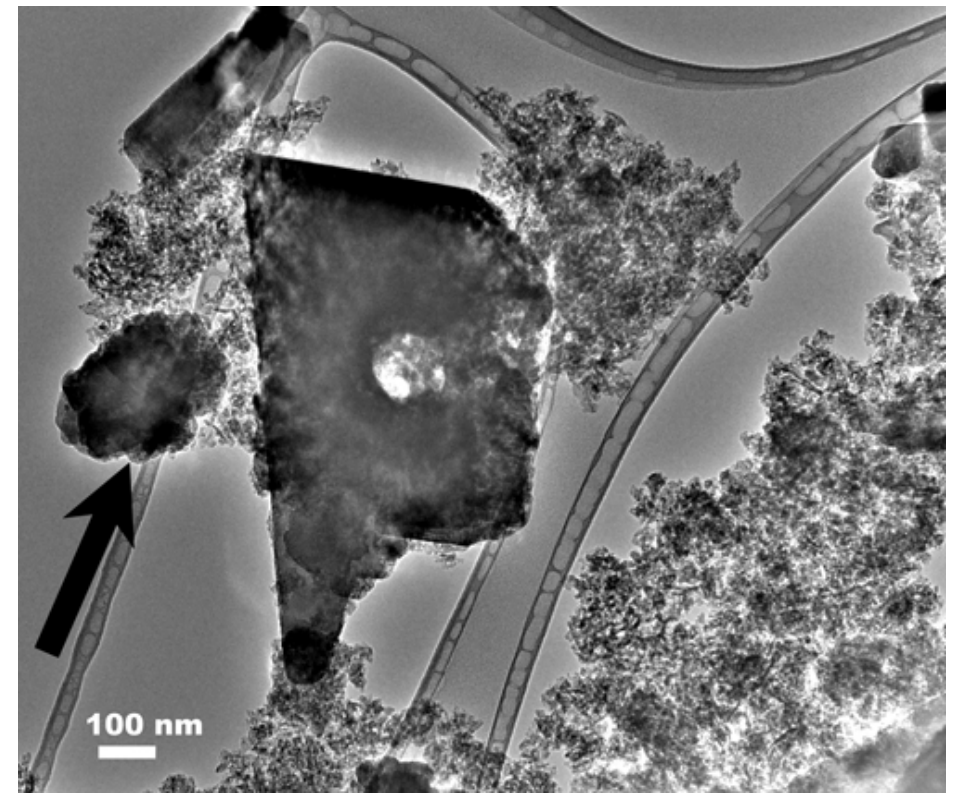

FIGURE 4

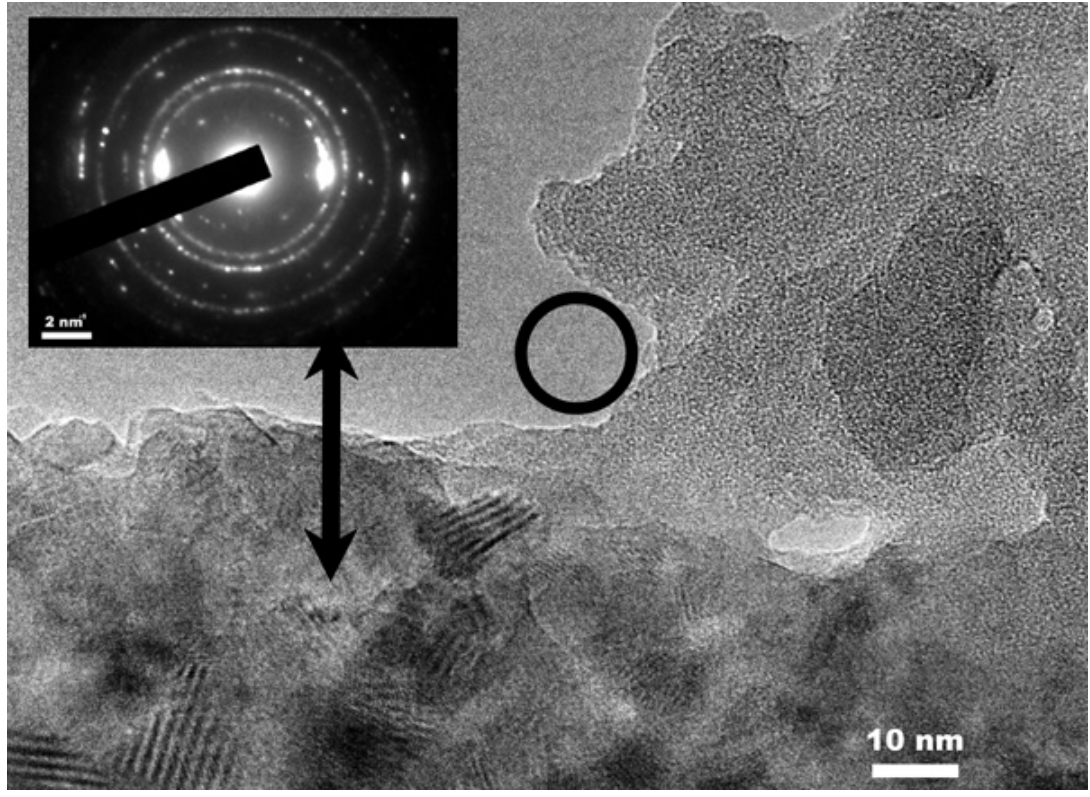

FIGURE 5

Thus, the carbonated C-S-H sample can be described, as a multitude of carbonate microcrystals ${ }^{\dagger}$, dispersed in a disordered matrix [35]. Calcite layers covering large areas

'We use the term "microcrystals" to design crystals with a size around hundreds of nanometers, to mark the difference with the previously defined embedded tobermorite "nanocrystals". 
of the residual C-S-H are observed as well (indicated by an arrow on Figure 4). Such layers of calcium carbonate covering the C-S-H structure have been previously reported $[24,36]$, and described as $\mathrm{CaCO}_{3}$ accumulated on the pores of tobermorite. This carbonate layer or film could eventually prevent the HD C-S-H from being fully carbonated (passivation layer, [37]), or it could, at least, slow down the carbonation process locally.

Small angle x-ray scattering

The same samples were also studied by Small Angle X-Ray Scattering [38,39].

Scattered intensities from both original and carbonated synthetic C-S-H and from original and carbonated hydrated cement are plotted on Figure 6 as a function of the modulus of the momentum transfer vector $q$. The similarity on the change of plot shape due to $\mathrm{CO}_{2}$ attack on both types of samples is remarkable and is attributed to similarity in the structural changes induced by the carbonation process. When analyzing the scattering curves starting from low $q$ values, all curves fit the exponential decay, in the range $q<$ $0.2 \mathrm{~nm}^{-1}$, corresponding to sizes greater than $30 \mathrm{~nm}$. Values of their exponents obtained by numerical fitting are indicated in Table 3 . For all samples, the slope decreased (in absolute values) with carbonation, from 3.16 to 2.83 for the $\mathrm{C}-\mathrm{S}-\mathrm{H}$ and from 3.07 to 2.92 for the hydrated cements. Thus, both samples increased the average roughness of the nanostructure, revealing a similar change in the roughness of the nanostructures. The disappearance of the needles and the precipitation of silica gel in the carbonated sample changed the fractal description from a surface fractal structure (|slope $\mid>3)$ to a volume fractal matrix $(\mid$ slope $\mid<3)$. This corresponds to the disappearance of the outer product which is characterized by a surface fractal [13]. 
TABLE 3

Comparing these features with the TEM images and $\mathrm{N}_{2}$ physisorption results, typical sizes of the structure of the original sample can be seen in Figure 2. That is, in the scale of the validity limit of the fractal approach $(>30 \mathrm{~nm})$, it behaves as a surface fractal and shows pore sizes of $11-13 \mathrm{~nm}$ as obtained by the BJH analysis.

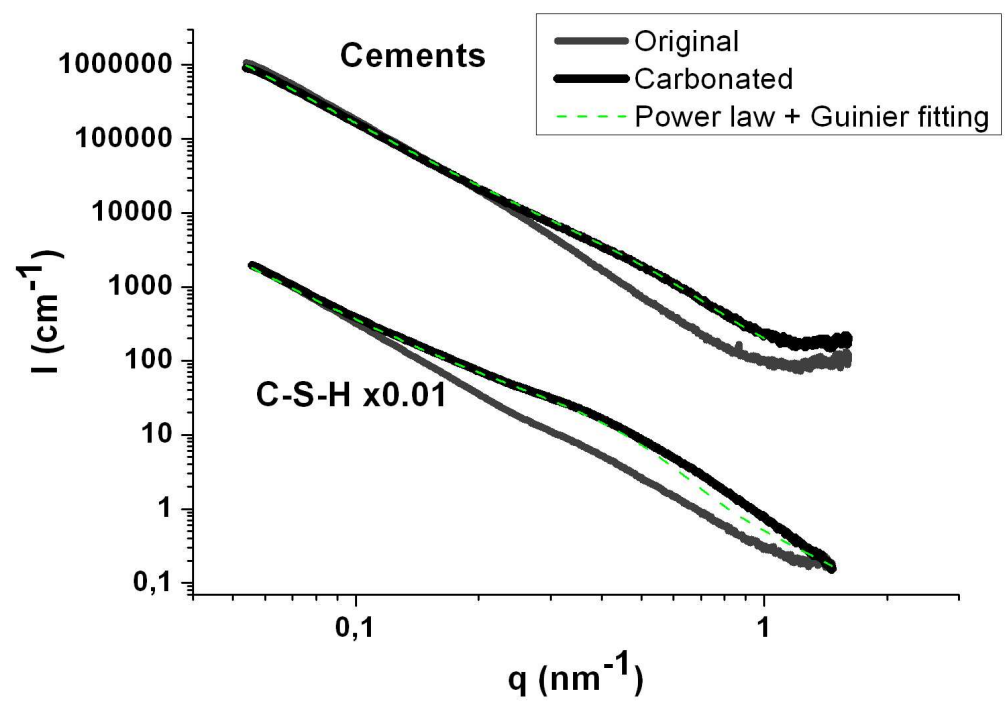

FIGURE 6

In the next q-range, the precipitation of the silica by-product also gave rise to a shoulder around $0.05 \mathrm{~nm}^{-1}$ in both carbonated C-S-H sample and carbonated hydrated cement. The calculated Guinier's radii are $5.01 \mathrm{~nm}$ and $4.37 \mathrm{~nm}$, respectively. The good fitting of both considered models (fractal and Guinier) with the experimental curves of both carbonated samples from lowest $q$ up to $q=0.75 \mathrm{~nm}^{-1}$ can be seen in Figure 6 . Given the relationship between a bulk sphere and Guinier's radius $\left(R=(5 / 3)^{1 / 2} R_{G}\right)$, the value of 
$\mathrm{R}_{\mathrm{G}}=5.01 \mathrm{~nm}$ for carbonated C-S-H yielded a characteristic radius $R \sim 6.5 \mathrm{~nm}$. A reference circle of $6.5 \mathrm{~nm}$ radius is drawn on Figure 5 to show the agreement between the results of the different techniques. Again, the mean pore diameter of $13 \mathrm{~nm}$ obtained by gas physisorption indicates that the pores are the scatterers in the silica gel structure, and not the particles (considering Babinet's principle).

Porod analyses of the high $q$-range of the scattering patterns were performed. The theoretical analysis shows that the scattering due to completely flat surfaces presents a slope $=-4$. Thus, in the Porod plots (lq4 vs. q), the existence of a plateau reveals the observation of a flat surface, typically associated to the interface of the structure, namely, the measurement of the specific surface area of the sample. These plots are traced on Figure 7, and no plateau was found for any of the samples except for the carbonated CS-H. No plateau was found for any of the samples except for the carbonated C-S-H. Horizontal black arrows on Figure 7 point at a very rough estimation of the values of Porod's constant, giving an assessment of the specific surface area for all the samples. Assuming a constant density for the solid phase of both the original and carbonated C-S$\mathrm{H}$ of $2.35 \mathrm{~g} / \mathrm{cm}^{3}$ (equal to those of tobermorite and jennite, and also similar to that of the silica), we found that specific surface area values changed from $214 \mathrm{~m}^{2} / \mathrm{g}$ to $458 \mathrm{~m}^{2} / \mathrm{g}$ in the synthetic $\mathrm{C}-\mathrm{S}-\mathrm{H}$ (Table 3 ) due to $\mathrm{CO}_{2}$ attack. This change corresponds to the behavior of the specific surface area obtained by gas physisorption, considering that the values obtained by nitrogen physisorption were underestimated due to the presence of non-porous phases, and assuming that there are closed porosities in the silica matrix or hidden porosity by the precipitated calcium carbonate [25]. Nevertheless, the specific surface area values obtained by SAXS can only be considered for qualitative comparison purposes given the rough estimation of Porod's constant, and they should not be 
considered as an accurate quantification of this parameter. As C-S-H is the most important contributor to the specific surface area of the hydrated cements, assessments of the values were also obtained at a constant skeleton density of $2.35 \mathrm{~g} / \mathrm{cm}^{3}$. The disappearance of the outer product and the formation of a porous silica matrix can also explain the increase in the specific surface area from $141 \mathrm{~m}^{2} / \mathrm{g}$ to $340 \mathrm{~m}^{2} / \mathrm{g}$.

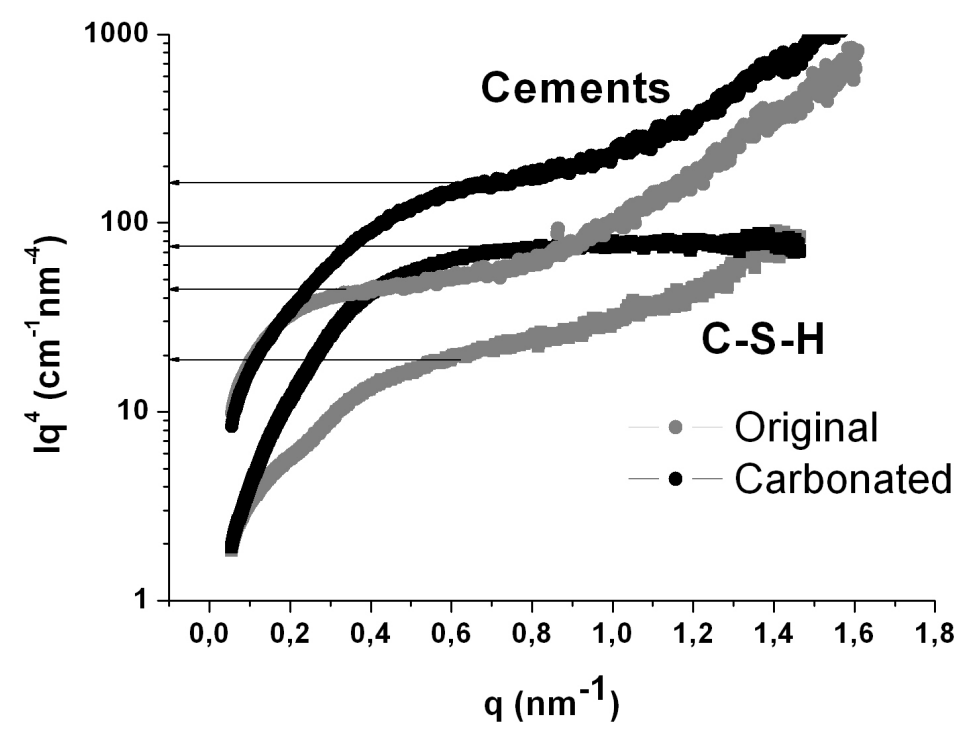

FIGURE 7

Finally, Debye's model [40] was also applied for analyzing the highest $q$-range of the scattering curves. This model was conceived for two-phase random media, in this case the solid skeleton and the porous space. The most important idea is that the autocorrelation function behaves as an exponential decay $e^{-r / a}$, with ' $a$ ' being the measure of the extension of the scatterers. Therefore, assuming this distribution, the derived scattered intensity is proportional to 


$$
I(q) \propto \frac{a^{3}}{\left(1+q^{2} a^{2}\right)^{2}} \quad \text { Eq. } 1
$$

From the linear region found in the Debye plot $I(q)^{-1 / 2}$ vs. $q^{2}$, Debye's characteristic length a can be derived. In Figure 8, Debye plots of the four samples are presented. Whereas Debye's model fits very well the data for carbonated synthetic $\mathrm{C}-\mathrm{S}-\mathrm{H}$, no acceptable fit was obtained for the original C-S-H and for both types of cement pastes, as the linear ranges were too small. Thus, only the structure of the carbonated synthetic C-S-H can bear a description as a two phase random medium, yielding a characteristic length of $4.2 \mathrm{~nm}$ for carbonated $\mathrm{C}-\mathrm{S}-\mathrm{H}$, which can be understood as the size of the elemental particles of the silica gel by-product, as the silica gel has already been described by Debye's model $[41,42]$.

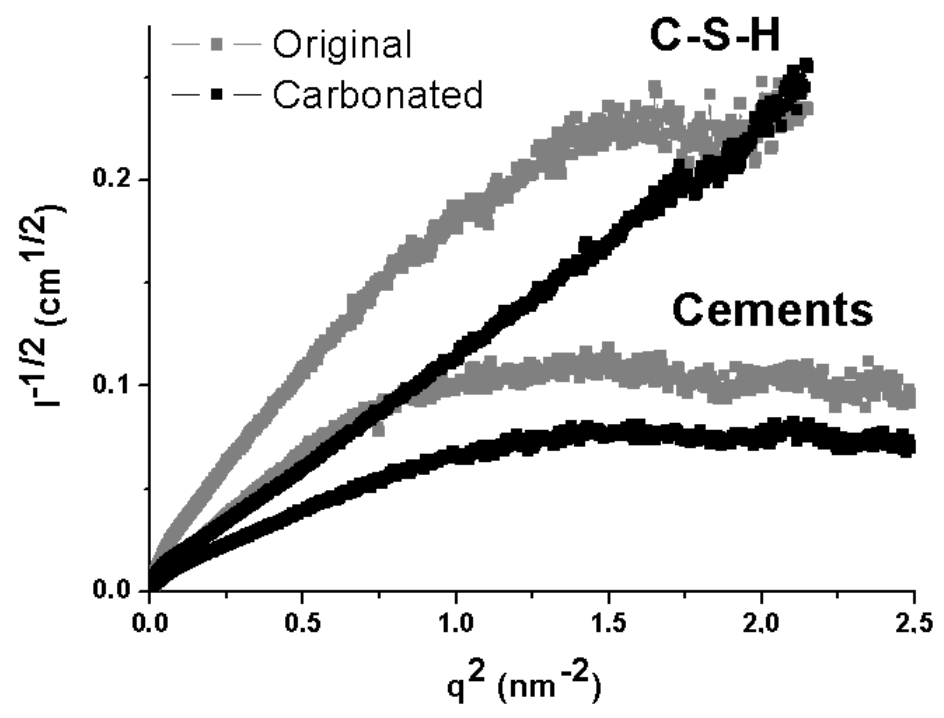

FIGURE 8 
Comparing these values with Guinier's model fitting, a hierarchical structural distribution of the particles can be inferred: Debye's model analyses smaller structural features (higher $q$ values), revealing therefore the properties of the elemental particles of the silica gel, which form clusters. The characteristic length of $13 \mathrm{~nm}$ resolved by Guinier's fitting corresponds to the size of the pores formed by the arrangement of the clusters.

\section{Conclusions}

A mixture of low density and high density synthetic $\mathrm{C}-\mathrm{S}-\mathrm{H}$ has been synthesized by the co-precipitation method. With a gentle aqueous $\mathrm{CO}_{2}$ attack at room temperature, low density C-S-H carbonated more easily and completely disappeared whereas some inner product remained, likely aided by the passivation effect of precipitated calcium carbonate, resulting in closing part of the porosities. In both synthetic C-S-H and hydrated Portland cements, carbonation led to the precipitation of silica gel by-products of identical structure, i.e. based on two hierarchical levels with a primary particle size of $4.2 \mathrm{~nm}$ and building clusters that agglomerated and formed pores of $13 \mathrm{~nm}$. Another prominent result of this study is the increase in the specific surface area revealed from SAXS which is due to the carbonation of the low density C-S-H and the precipitation of the silica gel carbonation product.

This study clearly points out the central role played by low density C-S-H's in the cement carbonation process through (1) their high reactivity, (2) the possible passivating effect of their Ca-carbonate products over high density C-S-H carbonation and (3) the formation of porous silica gel which will likely control the transport of $\mathrm{CO}_{2}$ aqueous species through the cement, the porosity of which being progressively filled with Ca-carbonates. 


\section{Acknowledgements}

We acknowledge the European Synchrotron Radiation Facility (ESRF) for provision of the synchrotron radiation facilities, and would like to especially thank Dr. F. Fauth for his assistance in using the beamline BM16. We also acknowledge the technical services of the Instituto de Ciencia de Materiales de Sevilla (CSIC-US) for its help with characterization measurements. This work was funded by the European Union through a Marie Curie grant within the GRASP project MRTN-CT-2006-035868. Finally, we would like to thank Dr. J. N. Rouzaud for his help with the TEM micrographies and Dr. Nicolas de la Rosa-Fox for discussion and his wise ideas.

\section{References}

1 Taylor HFW (1997) Cement Chemistry. Thomas Telford Publishing, London

2 Barlet-Gouédard V, Rimmelé G, Porcherie O, Quisel N, Desroches J (2009) Int. J. of Greenh Gas Control 3:206. doi:10.1016/j.ijggc.2008.07.005

3 Jaramillo P, Griffin WM, MCCoy ST (2009) Environ. Sci Technol 43:8027. doi:10.1021/es902006h

4 Rimmelé G, Varlet-Gouédard V, Porcherie O, Goffé B, Brunet F (2008) Cem. Concr. Res. 38:1038. doi:10.1016/j.cemconres.2008.03.022

5 See for example: GRASP project (http://www.grasp-co2.eu) or CO2Sink project (http://www.co2sink.org). Last visit: September 5 ${ }^{\text {th }}, 2010$.

6 Corvisier J, Brunet F, Fabbri A, Bernard S, Findling N, Rimmelé G, Barlet-Gouédard V, Beyssac O, Goffé B (2010) Eur. J. Mineral 22:63. doi:10.1127/09351221/2010/0022-1977

7 Scherer, G. W. (1999) Cem. Concr. Res. 29:1149-1157.

8 Feldman RF, Sereda PJ (1970) Eng. J. Can. 53:53

9 Jennings HM (2000) Cem Concr Res 30:101 
10 Jennings HM (2008) Cem Concr Res 38:275

11 Richardson IG (2004) Cem Concr Res 34:1733.

doi:10.1016/j.cemconres.2004.05.034

12 Frattini E, Chen SH, Baglioni P, Bellissent-Funel MC (2001) Physical Review E 64:020201

13 Popova A, Geoffroy G, Gartner EM, Lapp A (2002) J Am Ceram Socy 85:1303. doi:10.1111/j.1151-2916.2002.tb00264.x

14 Morales-Flórez V, Brunet F (2009) Molecular Simulation 35:1001. doi:10.1080/08927020903033117

15 Lloyd RR, Provis JL, Van Deventer JSJ (2009) J Mater Sci 44:620.

doi:10.1007/s20853-008-3078-z

16 Mazumder S, Sen D, Bahadur J, Klepp J, Rauch H, Teixeira J (2010) Phys Rev B 82:064203. doi:10.1103/PhysRevB.82.064203

17 Thomas JJ, Jennings HM, Allen AJ (1998) Cem Concr Res 28:897. doi:10.1016/S0008-8846(98)00049-0

18 Allen AJ, Thomas JJ, Jennings HM (2007), Nat Materials 6:311. doi:10.1038/nmat1871

19 Blinc R, Lahajnar G, Zummer S (1988) Phys Rev B 38:2873

20 Thomas JJ, Allen AJ, Jennings HM (2008) J Am Ceram Soc 91:3362

21 Skinner LB, Chae SR, Benmore CJ, Wenk HR, Monteiro PJM (2010) Phys Rev Lett 104:195502. doi:10.1103/PhysRevLett.104.195502

22 Manzano H, Ayuela A, Dolado JS (2007) Journal of Computer-Aided Materials Design 14:45. doi:10.1007/s10820-006-9030-0

23 Gonzalez-Teresa R, Morales-Florez V, Manzano H, Dolado JS (2010) Materiales de construccion 60:7. doi: 10.3989/mc.2010.57010

24 Häussler F, Palzer S, Eckart A, Hoell A (2002) Appl Phys A 74:S1124. doi:10.1007/s003390101202

25 Johannesson B, Utgennant P (2001) Cem Concr Res 31:925

26 Sugiyama D, Fujita T (2006) Cem Concr Res 36:227

27 Brunauer S, Emmett PH, Teller E (1938) J Am Chem Soc 60:309

28 Barret EP, Joyner LG, Halenda PP (1951) J Am Chem Soc 73:373

29 Image J 1.40g, By Wayne Rasband. National Institute of Health, USA. http://rsb.info.nih.gov/ij. Last visit: september 2010.

30 Sing KSW, Everett DH, Haul R, Moscou L, Pierotti RA, Rouquérol J, Siemeniewska T (1985) Pure \& Appl Chem 57:603. doi:10.1351/pac198557040603

31 Zhang X, Chang W, Zhang T, Ong CK (2000) J Am Ceram Soc 83:2600 
32 Viehland D, Li JF, Yuan LJ, Xu Z (1996) J Am Ceram Soc 79:1731

33 Xu Z, Viehland D (1996) Physical Review Letters 77:952.

doi:10.1103/PhysRevLett.77.952

34 American Mineralogist Crystal Structure Database. http://rruff.info, last visit:

December 2010.

35 Black L, Garbev K, Gee I (2008) Cem Concr Res 38:745

36 Siauciunas R, Rupsyté E, Kitrys S, Galeckas V (2004) Coll and Surf A 244:197

37 Béarat H, McKelvy MJ, Chizmeshya AV, Gormley D, Nunez R, Carpenter RW, Squires K (2006) Environ Sci Technol 40:4802. doi:10.1021/es0523340

38 Glatter O, Kratky O (1982) Small Angle X-Ray Scattering. Academic Press, London 39 Allen AJ, Thomas JT (2007) Cement and Concrete Reseach 37:319.

doi:10.1016/j.cemconres.2006.09.002

40 Debye P, Bueche AM (1949) J Appl Phys 20:518

41 Longman GW, Wignall GD, Hemming M, Dawkins JV (1974) Colloid \& Polymer Sci 252:298

42 De la Rosa-Fox N, Morales-Flórez V, Toledo-Fernández JA, Piñero M, Esquivias L, Keiderling U (2008) J Sol-Gel Sci and Tech 45:245. doi:10.1007/s10971-008-1686-3 


\section{TABLES AND FIGURES}

\section{CAPTIONS}

Table 1. Texture values of the synthetic $\mathrm{C}-\mathrm{S}-\mathrm{H}$ and hydrated cements obtained by nitrogen physisorption.

Table 2. Identified reflections by direct observation of the TEM image or by electron diffraction patterns; $($ - $)=$ no reflection was found for the corresponding interplanar distance.

Table 3. Data from the scattering curves plotted in Figure 6: the power law decay exponent $(\mathrm{m})$ and fractal dimension (Ds for surface fractals and Dv for volume fractals) governing the power law decay; the validity size range of this description is $r>30 \mathrm{~nm}$, except for carbonated C-S-H which is $\mathrm{r}>60 \mathrm{~nm}$; Guinier's radii from model fitting on the shoulder. ( - ) Guinier's or Debye's model could not be successfully fitted; specific surface area was obtained by estimating Porod's constant in Figure 7; Debye's characteristic length estimated by the fitting shown in Figure 8.

FIG. 1. Nitrogen physisorption isotherm cycles and pore size distributions (inset) for original and carbonated C-S-H.

FIG. 2. TEM micrograph of the original C-S-H. Tobermorite-like needles typical for Low Density C-S-H type can be observed as well as a disordered particulate mesoporous structure (High Density C-S-H). 
FIG. 3. Detailed view of the boundary of the HD C-S-H (original sample before carbonation). The embedded nanocrystals as well as Moire's patterns can be seen clearly. Inset: electron diffraction pattern of the same area; measured interplanar distances are listed in Table 2.

FIG. 4. TEM image of the carbonated C-S-H sample. Calcium carbonate microcrystals can be distinguished as well as films covering the porous matrix (pointed by the arrow). The degradation of the calcium carbonate microcrystals due to electron beam damage can be also observed; the circular hole was made in just five seconds by focusing the beam. Note that this beam damage was not observed on embedded calcium silicate nanocrystals (Figures 2 and 4).

FIG. 5. TEM image of carbonated C-S-H. Two different morphologies can be easily identified: the HD C-S-H with embedded nanocrystals, similar to that observed in the original sample (lower part of the image) and the silica gel resulting from C-S-H carbonation (upper right part). The circle shows the typical particle size of $13 \mathrm{~nm}$ obtained by the SAXS data treatment. Inset: Electron diffraction pattern focused on the disordered area indicated by the arrow.

FIG. 6. Log-log plot of the scattered intensities of the original and carbonated C-S-H and cement samples. Intensities for $\mathrm{C}-\mathrm{S}-\mathrm{H}$ have been multiplied by a factor of 0.01 for clarity. The fitting model is the sum of the power law decay and Guinier's model.

FIG. 7. Porod plot of the hydrated cements and C-S-H samples, original and carbonated. 
Values of the Porod constants and therefore of the specific surface areas are only considered as rough estimates due to the lack of a well-defined Porod region.

FIG. 8: Debye's plot for the four samples, cements and C-S-H, original and carbonated. The derived Debye's characteristic length of carbonated C-S-H is shown in Table 3. The linear region of each curve was used for derivation of Debye's characteristic length. 
TABLES

TABLE 1

\begin{tabular}{|c|c|c|c|}
\hline C-S-H & $\mathbf{S}_{\mathrm{BET}}\left(\mathbf{m}^{2} \mathbf{g}\right)$ & $\begin{array}{c}\text { Porous volume } \\
\left(\mathbf{c m}^{3} \mathbf{g}\right)\end{array}$ & $\begin{array}{c}\text { Mean pore size } \\
(\mathbf{n m})\end{array}$ \\
\hline Original & 95 & 0.52 & $11-13$ \\
\hline Attacked & 132 & 0.68 & 13 \\
\hline Hydrated cements & & & \\
\hline Original & 26 & 0.07 & $4.7 \mathrm{~nm}$ \\
\hline Attacked & 26 & 0.08 & $\begin{array}{l}1.9 \mathrm{~nm} \text { and } \\
2.7-4.7 \mathrm{~nm}\end{array}$ \\
\hline
\end{tabular}


TABLE 2

\begin{tabular}{|c|c|c|}
\hline Image & $\begin{array}{l}\text { Resolved distances } \\
\text { (1 Ångstrom = } 0.1 \mathrm{~nm} \text { ) }\end{array}$ & Tobermorite reflections \\
\hline $\begin{array}{r}\text { Embedded nanocrystals of the } \\
\text { original C-S-H } \\
\text { (Figure } 2)\end{array}$ & $\begin{array}{l}4.90 ; 2.56 ; 2.44 \\
2.43 ; 2.38 ; 2.34 \\
2.33 ; 2.29 ; 2.27\end{array}$ & 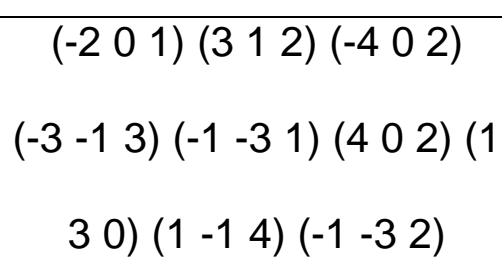 \\
\hline $\begin{array}{r}\text { Electron diffraction pattern } \\
\text { (Inset Figure 2) }\end{array}$ & $\begin{array}{c}3.47 ; 2.79 ; 2.50 \\
2.43 ; 1.75 ; 1.71 \\
1.39 ; 1.08\end{array}$ & 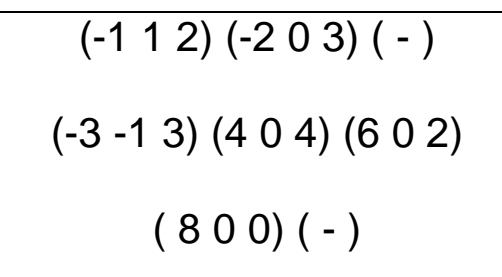 \\
\hline $\begin{array}{r}\text { Embedded nanocrystals in } \\
\text { carbonated C-S-H } \\
(\text { Figure 4) }\end{array}$ & $\begin{array}{l}3.49 ; 3.10 ; 2.99 \\
2.94 ; 2.89 .2 .87 \\
2.84 .2 .81 .2 .57 \\
2.50 ; 2.48 ; 2.46\end{array}$ & 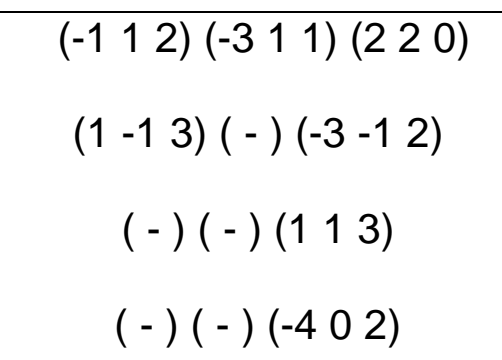 \\
\hline $\begin{array}{r}\text { Electron diffraction pattern } \\
\text { (Inset Figure 4) }\end{array}$ & $\begin{array}{l}5.88 ; 3.97 ; 3.77 \\
3.00 ; 2.78 ; 2.40 \\
2.08 ; 1.70 ; 1.51\end{array}$ & 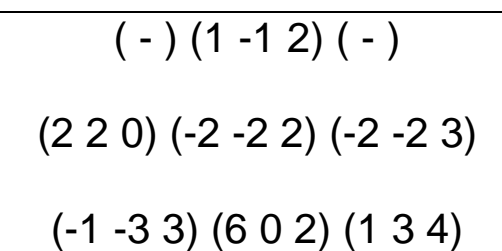 \\
\hline
\end{tabular}




\section{TABLE 3}

\begin{tabular}{cccccc} 
Sample & $\begin{array}{c}\text { Decay } \\
\text { exponent }\end{array}$ & $\begin{array}{c}\text { Fractal } \\
\text { dimension }\end{array}$ & $\begin{array}{c}\text { Guinier } \\
\text { radius } \\
(\mathrm{nm})\end{array}$ & $\begin{array}{c}\text { Specific } \\
\text { surface } \\
\text { area } \\
\left(\mathrm{m}^{2} / \mathrm{g}\right)\end{array}$ & $\begin{array}{c}\text { Debye's } \\
\text { length } \\
(\mathrm{nm})\end{array}$ \\
\hline $\begin{array}{c}\text { C-S-H original } \\
\text { C-S-H }\end{array}$ & 3.16 & $\mathrm{D}_{\mathrm{s}}=2.84$ & - & 214 & - \\
\hline $\begin{array}{c}\text { carbonated } \\
\text { Cement original }\end{array}$ & 2.83 & $\mathrm{D}_{\mathrm{v}}=2.83$ & 5.01 & 458 & -2 \\
\hline $\begin{array}{c}\text { Cement } \\
\text { carbonated }\end{array}$ & 2.07 & $\mathrm{D}_{\mathrm{s}}=2.93$ & - & 141 & - \\
\hline
\end{tabular}

